\title{
Quantitation of progenitor cell populations and growth factors after bone marrow aspirate concentration
}

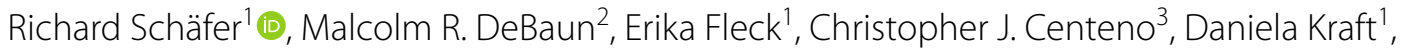 \\ Johannes Leibacher ${ }^{1}$, Karen Bieback ${ }^{4}$, Erhard Seifried ${ }^{1}$ and Jason L. Dragoo ${ }^{2^{*}}$
}

\begin{abstract}
Background: The number of Mesenchymal Stem/Stromal Cells (MSCs) in the human bone marrow (BM) is small compared to other cell types. BM aspirate concentration (BMAC) may be used to increase numbers of MSCs, but the composition of MSC subpopulations and growth factors after processing are unknown. The purpose of this study was to assess the enrichment of stem/progenitor cells and growth factors in BM aspirate by two different commercial concentration devices versus standard BM aspiration.

Methods: $120 \mathrm{~mL}$ of BM was aspirated from the iliac crest of 10 male donors. Each sample was processed simultaneously by either Emcyte GenesisCS ${ }^{\circledR}$ (Emcyte) or Harvest SmartPReP2 BMAC (Harvest) devices and compared to untreated BM aspirate. Samples were analyzed with multicolor flow cytometry for cellular viability and expression of stem/progenitor cells markers. Stem/progenitor cell content was verified by quantification of colony forming unit-fibroblasts (CFU-F). Platelet, red blood cell and total nucleated cell (TNC) content were determined using an automated hematology analyzer. Growth factors contents were analyzed with protein quantification assays. Statistical analyses were performed by ANOVA analysis of variance followed by Tukey's multiple comparison test or Wilcoxon matched-pairs signed rank test with $p<0.05$ for significance.
\end{abstract}

Results: Cell viability after processing was approximately $90 \%$ in all groups. Compared to control, both devices significantly enriched TNCs and platelets, as well as the CD45-CD73+ and CD45-CD73+CD90+ cell populations. Further, Harvest significantly concentrated CD45-CD10+, CD45-CD29+, CD45-CD90+, CD45CD105+, CD45-CD119+ cells, and CD45dimCD90+CD271+ MSCs, whereas Emcyte significantly enriched CD45dimCD44+CD271+MSCs. BM concentration also increased the numbers of CFU-F, platelet-derived growth factor, vascular endothelial growth factor, macrophage colony-stimulating factor, interleukin-1 b, VCAM-1 and total protein. Neither system concentrated red blood cells, hematopoietic stem cells or bone morphogenetic proteins.

Conclusion: This data could contribute to the development of BMAC quality control assays as both BMAC systems concentrated platelets, growth factors and non-hematopoietic stem cell subpopulations with distinct phenotypes without loss of cell viability when compared to unprocessed BM.

Keywords: Bone marrow aspirate concentrate, Bone marrow aspiration, BMAC, Growth factors, Mesenchymal stem cell, Stem cell, Stromal cell

\footnotetext{
*Correspondence: jdragoo@stanford.edu

2 Department of Orthopedic Surgery, Stanford University School

of Medicine, 450 Broadway, Redwood City, CA 94063, USA

Full list of author information is available at the end of the article
} 


\section{Background}

Human BM aspirate contains Mesenchymal Stem/Stromal Cells (MSCs) $[1,2]$, but the number of MSCs are low, estimated at $0.01-0.02 \%$ of the total cell volume [3]. Therefore, processing BM by BMAC may be clinically beneficial to increase the concentration of MSCs [4-6]. In recent years, the use of bone marrow aspirate concentrate (BMAC) has become an increasingly popular method of augmenting bone $[7,8]$ and cartilage regeneration [1] in orthopaedic surgery. BMAC is obtained through density gradient centrifugation of bone marrow (BM) typically aspirated from the iliac crest. It is currently approved by the United States Food and Drug Administration for clinical use as a means of acquiring progenitor cells and growth factors to promote healing in the orthopaedic patient [2].

A combination of different markers has been suggested to characterize MSCs present within BM, but especially in vitro expanded MSCs, e.g. CD45-, CD90+, CD73+, and CD271+ [5]. However, the exact phenotype of MSCs has not been agreed on yet due to the intrinsic heterogeneity of BM being comprised of different cell subsets and likely variable differentiation stages of the MSCs. Previous studies have reported considerable donordonor variation that increases the heterogeneity of MSC phenotypes [5]. Consequently, clinical trials employing MSCs are inconsistent, hampering their broad translation into clinical practice. It is imperative to compare BMAC produced by commercially available systems to better understand its therapeutic potential and to eventually establish a correlation between the progenitor cell composition, the concentration of biologic factors and clinical outcome.

The purpose of this study is to analyze and compare progenitor cell composition and growth factors in BMAC prepared from single donors and processed concurrently by two commercially available systems versus unprocessed marrow aspiration. We hypothesized that the BMAC systems will concentrate progenitor cell populations when compared to unprocessed $\mathrm{BM}$ without a meaningful loss of cell viability.

\section{Methods}

\section{Bone marrow aspirate collection and processing}

After ethical approval and informed consent was obtained, $120 \mathrm{~mL}$ (60 mL per iliac crest) of bone marrow aspirate (BMA) was harvested from the anterior iliac crests of 10 male adult human donors (age range $28-35$ years) using multiple puncture sites. Four $\mathrm{mL}$ of aspirate was withdrawn into a $60 \mathrm{~mL}$ syringe at each site into a syringe containing $1000 \mathrm{U} / \mathrm{mL}$ of heparin (SigmaAldrich, St. Louis, MO, USA). This process was repeated after the side-port Jamshidi needle was rotated 180 degrees and was withdrawn $1 \mathrm{~cm}$ until a total volume of $60 \mathrm{~mL} \mathrm{BM}$ was obtained. This process was then repeated using the contralateral iliac crest and then consolidated into one BM sample of $120 \mathrm{~mL}$ per donor. Donors with conditions that could potentially compromise BM quality, including history of hematologic malignancy, current chemotherapy, BM suppressive and anti-platelet medications, previous BMA, or acute illness where excluded from this study.

Under sterile laboratory conditions, the BMA was agitated with a shaker and was alloquated on a rotating basis into the two experimental groups, which totaled $55 \mathrm{~mL}$ aliquots for each BMAC system, i.e. Emcyte GenesisCS ${ }^{\circledR}$ (Fort Myers, FL, USA) and Harvest SmartPReP2 BMAC $^{\circledR}$ (Lakewood, CO, USA), and processed per the manufacturer's instructions.

Company representatives were present ensuring proper protocols were followed. $10 \mathrm{~mL}$ of BMA was randomly allocated into the unprocessed control group, and served as internal control for the respective donor/experiment. Each system was adjusted to produce $7 \mathrm{~mL}$ of BMAC. All samples were re-suspended in Alpha MEM media (Lonza, Basel, Switzerland) and 1\% Antibiotic-Antimycotic (Gibco, Grand Island, NY, USA) (7 mL BMAC in a total volume of $30 \mathrm{~mL} ; 10 \mathrm{~mL}$ BMA in a total volume of $15 \mathrm{~mL})$.

\section{Quantification of blood cells}

Red blood cells (RBCs), platelets and total nucleated cells (TNCs) were quantified in each group using the Sysmex XT-1800i Automated Hematology Analyzer (Kobe, Japan).

\section{Flow cytometry analyses}

Flow cytometry was performed to assess the impact of BMAC on important MSC populations [6-8]. Before flow cytometry analysis, BMA cells were washed with PBS and mononuclear cells were separated using Biocoll Separating Solution (Biochrom GmbH, Berlin, Germany) by centrifugation at $+4{ }^{\circ} \mathrm{C}$, without brake for $25 \mathrm{~min}$. at $400 \times g$. To detach cells possibly adherent to the tube, they were incubated with $0.25 \%$ trypsin/EDTA (Gibco) for 5 min. at $+37{ }^{\circ} \mathrm{C}$. After trypsin inactivation with phosphate buffered saline (PBS-DPBS; Gibco) $+10 \%$ fetal calf serum (PAN-Biotech $\mathrm{GmbH}$, Aidenbach, Germany) they were added to the BMA samples prior to Biocoll separation. RBC lysis was performed by incubating the cells for 25 min. at $+37^{\circ} \mathrm{C}$ with Red Blood Cell Lysing Buffer (Sigma, Steinheim, Germany). After an additional washing step with PBS, multicolor staining was performed at the manufacturer's recommended concentrations of specific primary antibodies or isotype controls. Non-labeled primary antibodies were detected by secondary antibody, 
goat-anti mouse-PE after incubation on ice for $20 \mathrm{~min}$ (Table 1). Via-Probe ${ }^{\mathrm{TM}}$-7AAD solution (BD Biosciences San Diego, CA, USA) was added to determine cell viability. To identify progenitor cells and MSC subpopulations we applied combinations of markers that are commonly accepted as being either present or absent on MSCs ("mandatory markers"). In addition, as a novel approach, we tested the co-expression of markers that have been described to identify MSC subpopulations ("optional markers") (Table 1). Flow cytometry was performed using the LSRFortessa ${ }^{\mathrm{TM}}$ cell analyzer (BD Biosciences) and data were analyzed using FACSDiva ${ }^{\mathrm{TM}}$ software (BD Biosciences). In order to detect rare stem cell subpopulations for each sample $1 \times 10^{6}$ events were analyzed. For gating strategy see Additional file 1: Figure S1.

\section{Colony-forming unit-fibroblasts (CFU-F) Assay}

To functionally assess non-hematopoietic stem/progenitor cell content CFU-F assays were performed. BMAC and control cell suspensions were applied to Biocoll Separating Solution (Biochrom $\mathrm{GmbH}$ ) and separated by density gradient centrifugation without break at $400 \times g$ for $20 \mathrm{~min}$. Mononuclear cells were washed twice with PBS followed by centrifugation at $400 \times g$ for $5 \mathrm{~min}$. The cell pellets were re-suspended in Alpha MEM (Lonza) supplemented with $10 \%$ human platelet lysate (German Red Cross Blood Service Baden-Württemberg-Hessen gGmbH, Frankfurt, Germany), 1x Gibco ${ }^{\circledR}$ Antibiotic-Antimycotic (Life Technologies, NY, USA), 2 IU/mL Heparin-Natrium 5000 (Ratiopharm, Ulm, Germany) and seeded at a density of $5 \times 10^{5}$ cells per well into 6 well plates (Thermo Fischer Scientific Nunc A/S, Roskilde, Denmark). Cells were incubated at $37{ }^{\circ} \mathrm{C}$ with $5 \% \mathrm{CO}_{2}$. The media was changed every 3 days, and the cultures were evaluated after 10 days microscopically. CFU-F were defined as a minimum of 50 cells per CFU-F. Colonies were counted in replicates and subsequently compared as mean data for each condition and donor.

\section{Enzyme-linked immunosorbent assay (ELISA)}

For quantification of growth factors and protein content in the cells, mononuclear cells from control and both BMAC groups, after Biocoll separation, were lysed (3 freeze/thaw cycles), and the lysate supernatants

Table 1 Antibodies used for flow cytometry and rationale for marker selection

\begin{tabular}{|c|c|c|c|c|c|}
\hline \multirow[b]{2}{*}{ Specific antibody } & \multirow[b]{2}{*}{ Fluoro-chrome } & \multirow[b]{2}{*}{ Clone } & \multirow[b]{2}{*}{ Manufacturer } & \multicolumn{2}{|l|}{ Rationale for analysis } \\
\hline & & & & $\begin{array}{l}\text { Mandatory } \\
\text { Marker presence/absence } \\
\text { identify MSCs (include "ISCT } \\
\text { markers") }\end{array}$ & $\begin{array}{l}\text { Optional } \\
\text { Marker presence refine MSC } \\
\text { subpopulations phenotypes }\end{array}$ \\
\hline Anti-human CD29 & $\mathrm{PE}$ & MAR4 & BD Biosciences & $\begin{array}{l}\text { Present in vitro (ISCT consen- } \\
\text { sus) [9] }\end{array}$ & \\
\hline Anti-human CD44 & $\mathrm{APC}$ & DB105 & $\begin{array}{l}\text { Miltenyi Biotec } \\
\text { (Bergisch-Gladbach, Germany) }\end{array}$ & Present in vitro [5] & \\
\hline Anti-human CD73 & APC & AD2 & $\begin{array}{l}\text { Biolegend } \\
\text { (San Diego, CA, USA) }\end{array}$ & $\begin{array}{l}\text { Present in vitro (ISCT consen- } \\
\text { sus) and in vivo }[4,5,28]\end{array}$ & \\
\hline Anti-human CD90 & APC & $5 E 10$ & BD Biosciences & Present in vitro (ISCT consen- & \\
\hline Anti-human CD90 & $\mathrm{PE}$ & $5 E 10$ & BD Biosciences & sus) and in vivo $[5,6,28]$ & \\
\hline Anti-human CD105 & purified & 266 & BD Bioscience & $\begin{array}{l}\text { Present in vitro (ISCT consen- } \\
\text { sus) [28] }\end{array}$ & \\
\hline Anti-human CD34 & FITC & 581 & $\begin{array}{l}\text { Molecular Probes Life Tech- } \\
\text { nologies } \\
\text { (Carlsbad, CA, USA) }\end{array}$ & $\begin{array}{l}\text { Absent in vitro (ISCT consen- } \\
\text { sus) [28]; identifies HSC }\end{array}$ & \\
\hline Anti-human CD45 & FITC & $\mathrm{HI} 30$ & BD Biosciences & $\begin{array}{l}\text { Absent in vitro (ISCT consen- } \\
\text { sus) [28]; identifies WBC and } \\
\text { HSC }\end{array}$ & \\
\hline Anti-human CD10 & PE & HI10A & BD Biosciences & & In vitro and in vivo $[4,5]$ \\
\hline Anti-human CD119 & $\mathrm{PE}$ & GIR-208 & BD Biosciences & & In vitro [5] \\
\hline Anti-human CD271 & $P E$ & ME20.4-1.H4 & Miltenyi Biotec & & In vitro and in vivo [4-6] \\
\hline Anti-human GD2 & purified & 14.G2A & BD Bioscience & & In vitro and in vivo $[4,5]$ \\
\hline Goat anti-mouse & PE & Polyclonal & BD Bioscience & & \\
\hline $\lg G 1$ & FITC & MOPC-21 & BD Biosciences & & \\
\hline $\lg G 1$ & $P E$ & MOPC-21 & BD Biosciences & & \\
\hline $\operatorname{lgG1}$ & $\mathrm{APC}$ & MOPC-21 & BD Biosciences & & \\
\hline
\end{tabular}


were then analyzed using the Quantibody-Array QAHBMA-1000-2 (RayBiotech, Norcross, GA, USA) and ELISA assays (Peprotech, Hamburg, Germany) following the manufacturer's instructions.

\section{Statistical analyses}

In order to account for donor-to-donor variability and to achieve data comparability between flow cytometry experiments, the percentages of viable antigen(s) positive/negative cells of the BMAC groups were divided by the percentages of the corresponding controls for each experiment. The calculated ratios represent the specific fold changes for each tested marker, or marker combination, of BMAC compared to the respective internal control (single donor).

To get an estimate about the respective cell numbers, percentages of subpopulations related to the recorded viable cell counts are presented in Additional file 2: Table S1.

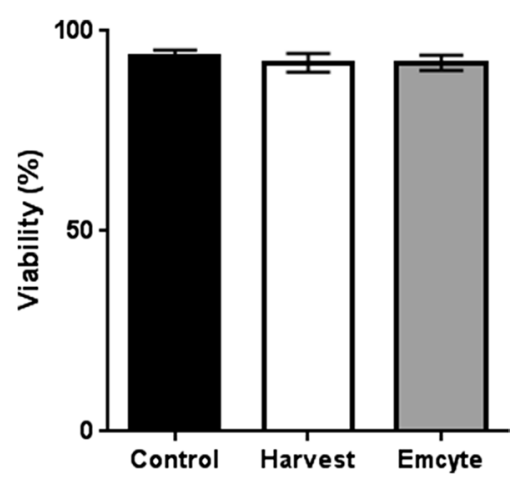

Fig. 1 Cell viability in both BMAC groups and controls. $N=10$ donors; ANOVA analysis of variance; error bars: standard error of means (SEM)
The statistical analyses were performed by ANOVA analysis of variance followed by Tukey's multiple comparison test, or Wilcoxon matched-pairs signed rank test using GraphPad Prism (La Jolla, CA, USA). Differences were considered significant when $\mathrm{p}<0.05$. Presented data for blood cell counts, CFU-F and growth factors were calculated of original non-diluted samples based on analyses of diluted samples considering respective sample dilutions (both BMAC: $4.29 \times$, controls: $1.50 \times$ ) to quantify and compare actual contents.

\section{Results}

Mean cell viability after processing was similar for unprocessed controls (91.57\%), Harvest (89.71\%) and Emcyte (92.29\%) systems ( $\mathrm{p}>0.05)$ (Fig. 1).

Concentrations of TNCs were significantly higher in both BMAC groups compared to controls indicating an approx. tenfold concentration (Harvest: $235.11 \times 10^{3}$ / $\mu \mathrm{L}, \quad \mathrm{p}=0.0472 ; \quad$ Emcyte: $265.28 \times 10^{3} / \mu \mathrm{L}, \quad \mathrm{p}=0.0307$; control: $24.49 \times 10^{3} / \mu \mathrm{L}$ ) (Fig. 2a). Both BMAC groups concentrated platelets (Harvest: $627.77 \times 10^{3} / \mu \mathrm{L}$, $\mathrm{p}=0.0205$; Emcyte: $802.23 \times 10^{3} / \mu \mathrm{L}, \mathrm{p}=0.0075$; control: $\left.100 \times 10^{3} / \mu \mathrm{L}\right)($ Fig. $2 \mathrm{~b})$ but depleted red blood cells (Harvest: $2.36 \times 10^{6} / \mu \mathrm{L}, \mathrm{p}=0.0016$; Emcyte: $1.57 \times 10^{6} / \mu \mathrm{L}$, $\mathrm{p}=0.0008$; control: $\left.5.99 \times 10^{6} / \mu \mathrm{L}\right)$ (Fig. $2 \mathrm{c}$ ).

Overall, hematopoietic cell $(\mathrm{CD} 45+)$ content was lower in both BMAC groups compared to the unprocessed control (Harvest: 0.86 fold vs control, $\mathrm{p}=0.5781$; Emcyte: 0.83 fold vs control, $p=0.375$ ) (Fig. 3a). Specifically, CD $45+29+$ cells were depleted (Harvest: 0.99 fold vs control, $p=0.9375$; Emcyte: 0.75 fold vs control, $\mathrm{p}=0.0469$ ) (Fig. 3b). Interestingly, the content of distinct CD45+cell populations was enriched after BM aspirate concentration, i.e. CD45+73+ (Harvest: 2.71 fold vs control, $\mathrm{p}=0.0313$; Emcyte: 2.13 vs control, $\mathrm{p}=0.1563), \mathrm{CD} 45+90+($ Harvest: 2.61 fold vs control, $\mathrm{p}=0.0781$; Emcyte: 2.74 vs control, $\mathrm{p}=0.0469$ ),
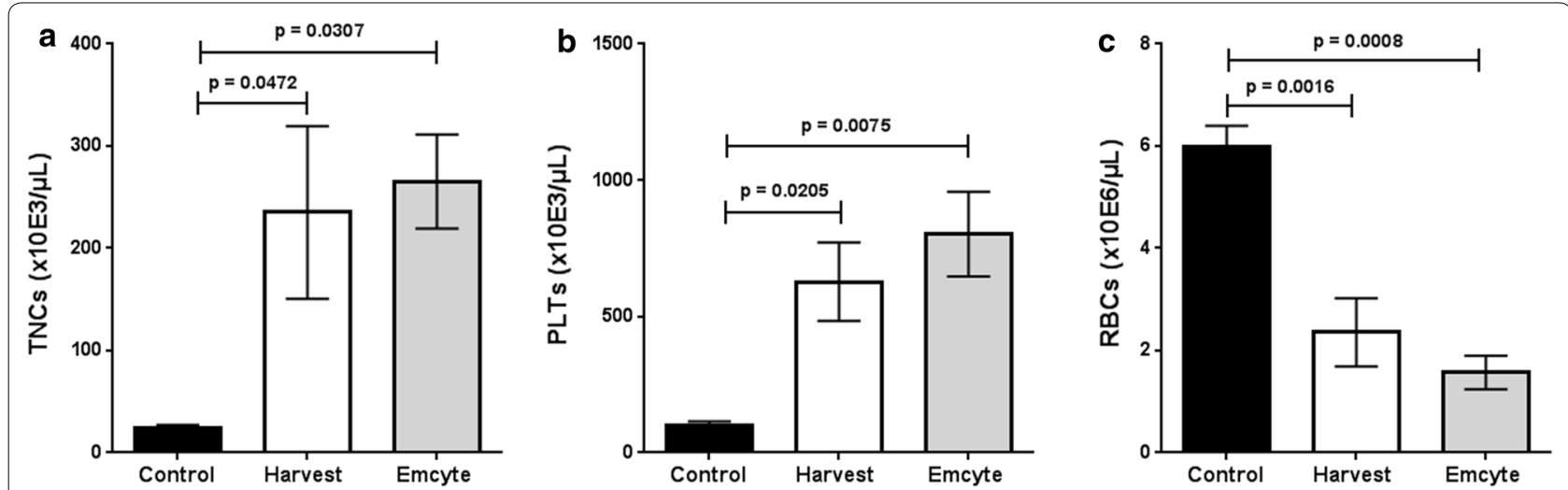

Fig. 2 Blood cell counts in both BMAC groups and controls. $\mathrm{N}=3$ donors; ANOVA analysis of variance; error bars: SEM 


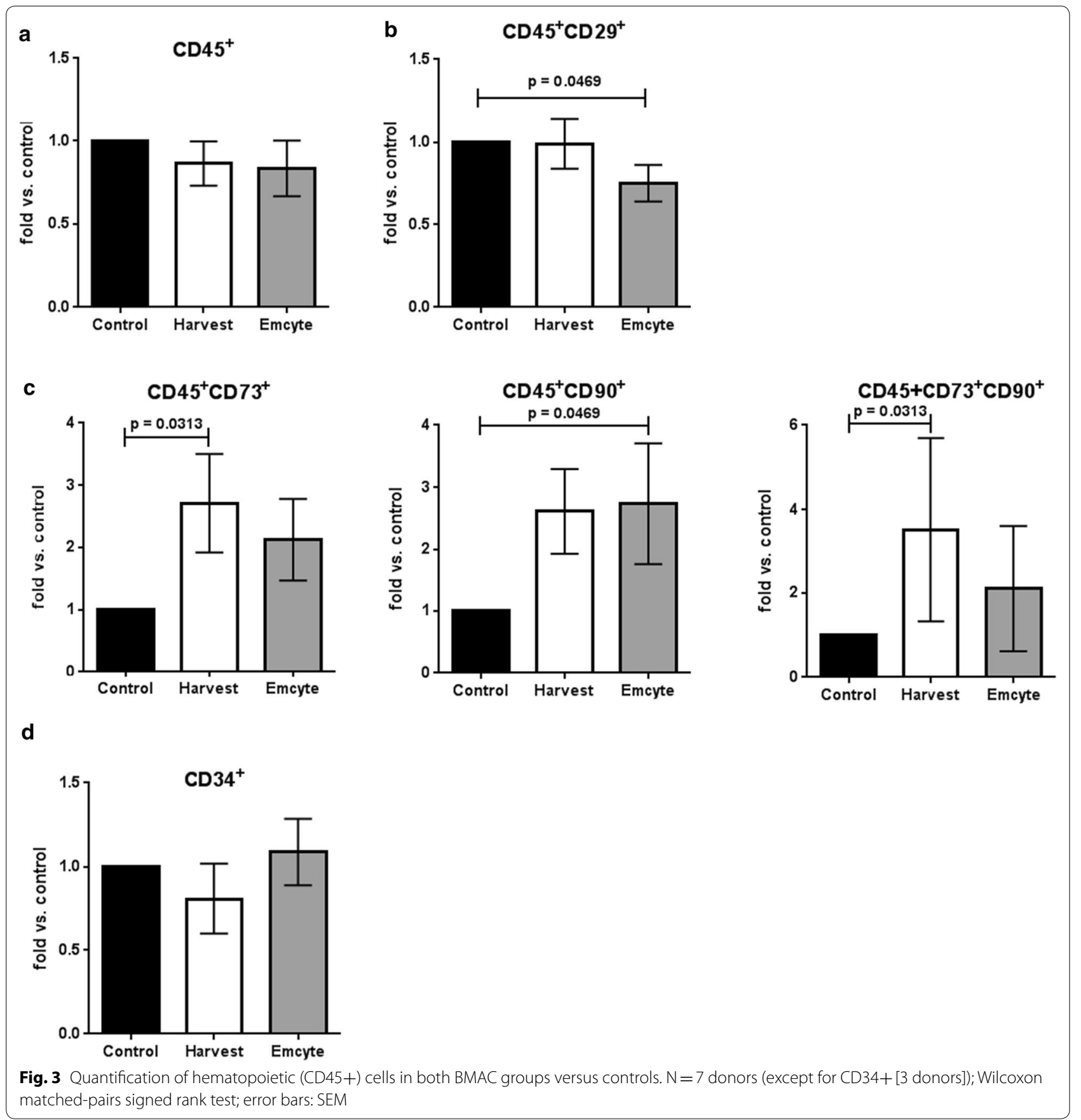

and CD45+73+CD90+(Harvest: 3.51 fold vs control, $\mathrm{p}=0.0313$; Emcyte: 2.11 vs control, $\mathrm{p}=0.0781$ ) cells (Fig. 3c). Notably, both BMAC groups did not concentrate CD34+ hematopoietic progenitor cells (Harvest: 0.809 fold vs control, $\mathrm{p}=0.71$; Emcyte: 1.088 vs control, $\mathrm{p}=0.93$ ) (Fig. 3d).

In contrast, the BMAC systems enriched distinct sets of CD45- and CD45dim non-hematopoietic progenitor subpopulations. Specifically, both devices significantly enriched CD45-CD73+ (Harvest: 9.2 fold vs control, $\mathrm{p}=0.0313$; Emcyte: 11.9 fold vs control, $\mathrm{p}=0.0469)$ and CD 45-CD73+CD90+ (Harvest: 12.9 fold vs control, $\mathrm{p}=0.0156$; Emcyte: 7.8 vs control, $\mathrm{p}=0.0469)$ cells.

Moreover, Harvest contained significantly more CD45-CD10+(3.6 fold vs control, $\mathrm{p}=0.0156)$, CD45-CD29+(1.6 fold vs control, $\mathrm{p}=0.0156)$, CD45-CD90+(14.8 fold vs control, $\mathrm{p}=0.0156)$, CD45-CD105+(8.8 fold vs control, $\mathrm{p}=0.0469)$, 

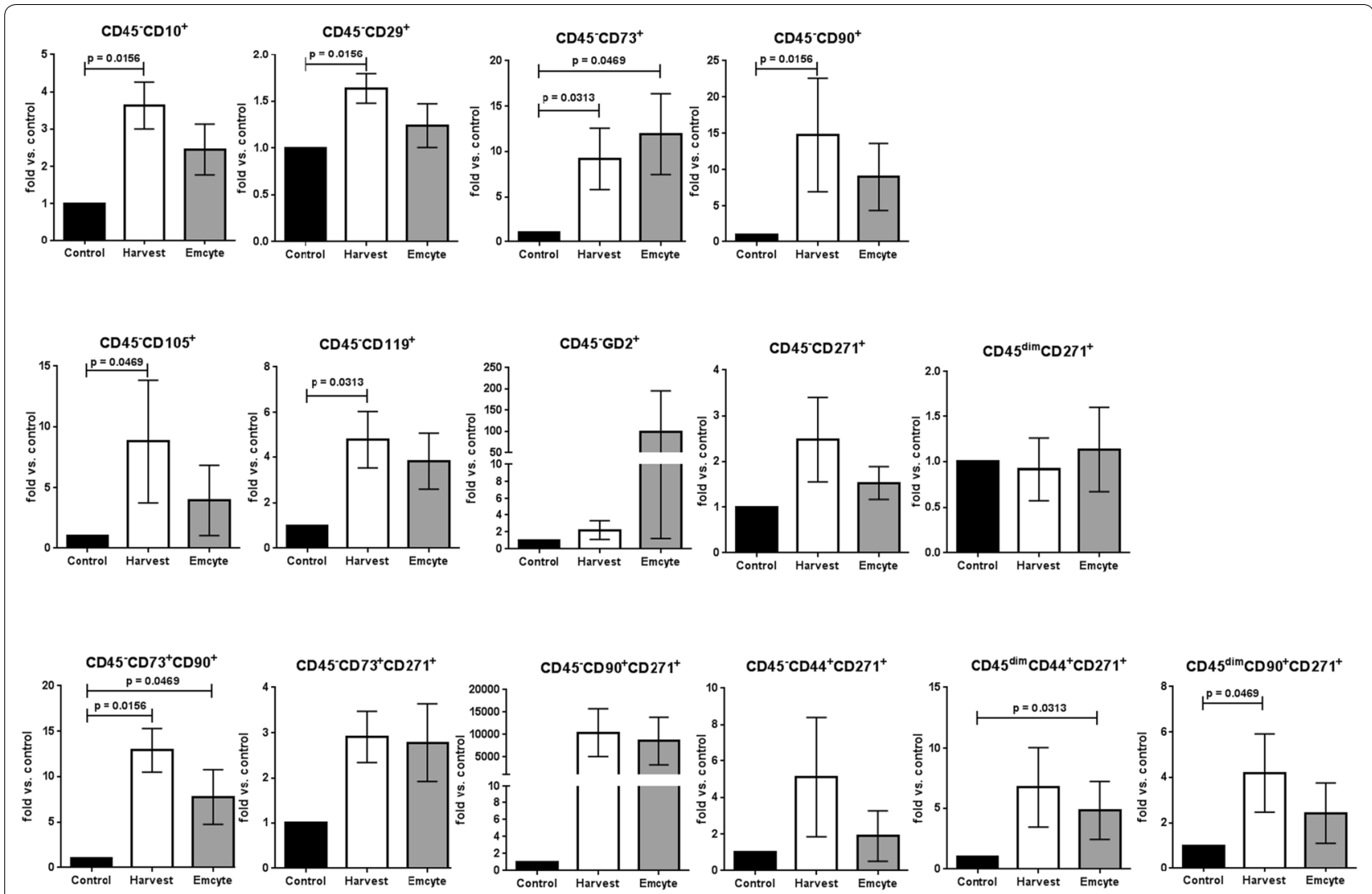

Fig. 4 Comparison of MSC subpopulations (CD45-/dim) detected in both BMAC groups versus controls; $N=7$ donors (except for CD45CD73+CD271 [4 donors], CD45-CD90+CD271 [5 donors], CD45-CD44+CD271 [5 donors], and CD45dimCD44+ CD271 [6 donors]); Wilcoxon matched-pairs signed rank test; error bars: SEM

CD45-CD119+(4.8 fold vs control, $\mathrm{p}=0.0313)$ cells, and CD45dimCD90+CD271+(4.2 fold vs control, $\mathrm{p}=0.0469)$ MSCs. Emcyte significantly enriched CD45dimCD44+CD271+(4.9 fold vs control, $\mathrm{p}=0.0313$ ) MSCs (Fig. 4).

In addition to stem/progenitor cell phenotype analyses by flow cytometry, the non-hematopoietic stem cell content was assessed by CFU-F assay. We observed for both BMAC a donor-depending non-hematopoietic

Table 2 Non-hematopoietic progenitor cells content in both BMAC groups and controls assessed by CFU-F assay

\begin{tabular}{|c|c|c|c|c|c|}
\hline & \multirow{2}{*}{$\begin{array}{l}\text { Control } \\
\text { CFU-F }\end{array}$} & \multicolumn{2}{|l|}{ Harvest } & \multicolumn{2}{|l|}{ Emcyte } \\
\hline & & CFU-F & $\begin{array}{l}\text { Enrichment } \\
\text { factor }\end{array}$ & $\overline{\mathrm{CFU}-\mathrm{F}}$ & $\begin{array}{l}\text { Enrichment } \\
\text { factor }\end{array}$ \\
\hline Donor 1 & 127.08 & 407.98 & 4.43 & 1970.65 & 21.15 \\
\hline Donor 2 & 88.23 & 1758.86 & 19.93 & 3638.99 & 41.24 \\
\hline Donor 3 & 393.32 & 8194.07 & 22.47 & 9766.19 & 26.44 \\
\hline
\end{tabular}

CFU-F: CFU-F total calculated per $\mathrm{mL}$ of original, non-diluted sample; enrichment factor: BMAC CFU-F compared to control CFU-F; data are presented as means per donor $(\mathrm{N}=3)$ progenitor cells enrichment between 4.4 and 41.2 fold (Table 2).

ELISA growth factor analysis of the cell lysates illustrated considerable variability between donors. Both Harvest and Emcyte concentrated platelet-derived growth factor (PDGF)-BB, vascular endothelial growth factor (VEGF), macrophage colony-stimulating factor (M-CSF), interleukin (IL)-1b, VCAM-1, osteoactivin and total protein compared to controls (Fig. 5). BMPs $-2,-4,-6,-7$ and -9 were not detectable in most samples.

\section{Discussion}

Both commercial BMAC systems significantly concentrated MSC populations, platelets and growth factors, but not hematopoietic progenitor cells, compared to untreated marrow aspirate from single donors. This is an important finding as the number of MSCs in BM is small, estimated at $0.01-0.02 \%$, compared to other cell types. Our data corroborate other studies showing BMAC systems are effective in concentrating MSCs $[9,10]$. 


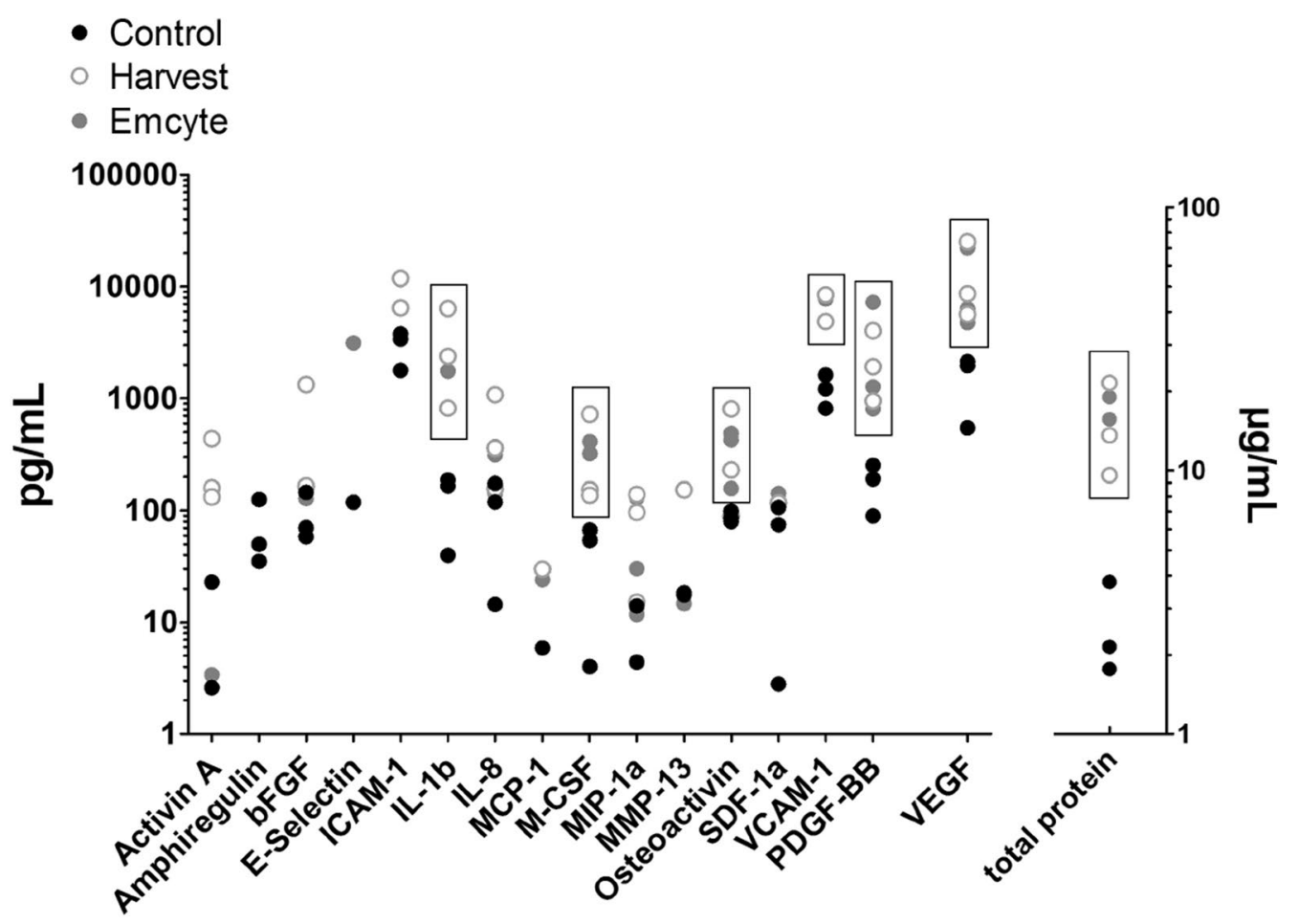

Fig. 5 Quantification of growth factors in MNC cell lysates of both BMAC groups and controls. $N=3$ donors; boxes highlight factors enriched by BMAC compared to controls. Points represent mean values for each donor

Despite enrichment of biologic factors, the question remains as to whether higher MSC content in BMAC leads to improved clinical efficacy. Hernigou et al. [11] illustrated that concentrating BMA could increase the progenitor cell population from $612 \pm 134$ progenitor cells $/ \mathrm{cm}^{3}$ before concentration to an average of $2579 \pm 1121$ progenitor cells $/ \mathrm{cm}^{3}$ after processing. In their study of patients with atrophic non-unions of the tibia who received percutaneous BMAC injections, the volume of mineralization of the fracture callus at 4 months was directly related to the number of progenitor cells in the original injection, providing evidence that the efficacy of BM aspirations for fracture healing can be enhanced with BMAC [3]. Bony union was achieved in $88 \%$ of their patients at 4 months following the procedure, and they concluded that BMAC was effective as a single stage procedure to treat atrophic tibial non-unions without the risk of complications related to in vitro expansion.

Hernigou et al. [3] also illustrated that fracture healing efficiency was associated with higher CFU-F numbers, a functional assay correlated to the clone forming capacity of the cells, in BMAC. Although our study did show an increase in CFU-Fs in both BMAC groups, the previous literature suggests that CFU-F quantification alone might not be sufficient to assess BMAC's therapeutic potential [12]. Moreover, the CFU-F assay cannot provide timely results as it requires several days ex vivo culture time.

BMAC may also promote tissue healing by delivering platelets as well as non-cellular growth factors [2, $5,11,13-15]$. Perhaps one of the more notable findings in this study is that BMAC increases concentrations of PDGF-BB and VEGF in mononuclear cells. PDGF-BB is a potent recruiter of cells crucial to musculoskeletal tissue repair, including MSCs, osteogenic cells and tenocytes. Additionally, it up regulates angiogenesis thereby initiating a cascade of bone and soft tissue repair mechanisms in the presence of injury [16]. VEGF belongs to a subset of the PGDF family and serves as a potent initiator of angiogenesis in response to injury [17]. As we analyzed the growth factors from the lysates of the mononuclear cell fraction we hypothesize that the higher growth factors concentrations we observed in BMAC were related to the higher numbers of progenitor cells rather than platelets contents. Yet, it may be reasonable to assume that both cell types contribute synergistically as they have been shown to carry such proteins [18].

Both BMAC technologies concentrated TNCs but did not increase the overall content of hematopoietic cells 
(CD45+). BMAC significantly reduced (CD45+CD29+) but also enriched (CD45+73+, CD45+90+, $\mathrm{CD} 45+73+\mathrm{CD} 90+)$ distinct subsets expressing markers that have been detected on a great variety of immune cells such as T cells, B cells, dendritic cells, and monocytes as well as on hematopoietic progenitor cells [19, 20].

Yet, to date, the effects of immune cell subset depletion or concentration by BMAC on tissue regeneration remains unclear. Additionally, the clinical relevance of specific significantly enriched CD45- (progenitor) subpopulations CD45-CD10+, CD45-CD29+, CD45-CD73+, CD45-CD90+, CD45-CD105+, CD45-CD119+, CD45-CD73+CD90+, CD45dimCD44+CD271+, and CD45dimCD90+CD271+is unknown, especially with regards to augmenting bone and/or cartilage regeneration. CD45-CD10+, CD45-CD29+, CD45-CD119+ and CD45-CD73+CD90+ phenotypes are part of both endothelial cell and MSC marker profiles [5], but their concrete relevance to regenerative medicine is also unknown. However, the increase of CFU-F supports the MSC enrichment in both BMAC groups, and the marker combination CD45dimCD90+CD271+ specifically characterizes highly clonogenic multipotent MSCs with significant osteogenic differentiation capacity [21, 22]. This specific subpopulation was concentrated in BMAC, providing further proof of concept for its clinical use. Yet, it has to be noted that the observed increase of CD45dimCD90+CD271+events detected by flow cytometry reflects the relationship to non-concentrated control but not absolute cell numbers. This is of particular importance as the frequency of these cells in the BM is very low, i.e. proximate to the flow cytometry detection limit. Despite this understanding, it is unclear if a greater presence of this subpopulation is relevant for clinical efficacy, as others have shown that platelets and trophic factors may also contribute to BMAC's therapeutic effect.

Previous studies have illustrated that BMAC application resulted in significant bone and cartilage healing in both animal models and human clinical trials [2, 3, 23]. In a recent review investigating the role of BMAC in animal long bone healing, $100 \%$ of the reviewed 35 manuscripts illustrated a significant increase in radiographic bone formation in the BMAC group, while $90 \%$ showed significant early bone healing on histological analysis and $78 \%$ showed increased torsional stiffness when compared to controls [24]. In a prospective non-randomized human clinical trial, patients with large patellofemoral chondral lesions showed significantly improved clinical outcome scores at a minimum 3 year follow-up after treatment with autologous BMAC implanted into the chondral defect which was comparable to patients treated with matrix-induced autologous chondrocyte implantation [25]. In another study, patients with chondral lesions treated with BMAC plus a collagen scaffold experienced significant clinical improvement in all clinical scores compared to preoperative values, complete filling of the defect in $80 \%$ and hyaline like cartilage regeneration on histological analysis [26]. However, there is currently a lack of consensus for or against the use of a scaffold coupled with BMAC for clinical applications [27].

\section{Conclusion}

Our data could contribute to the development of BMAC quality control assays as both commercial BMAC systems significantly concentrate MSC populations, platelets and growth factors, but not hematopoietic progenitor cells, compared to un-concentrated marrow aspirate. Clinical trials will be necessary to correlate specific MSC subpopulations and growth factors with therapeutic efficacy.

\section{Additional files}

Additional file 1: Figure 1. Multicolor flow cytometry gating strategy to identify living cells (7-AAD negative), followed by identification of hematopoietic (CD45+) from non-hematopoietic (CD45-) cells with subsequent analysis (representative example). Note differences of viable CD45-CD73+CD90+ MSCs located in the upper right quadrants (asterisks) between BMAC and control.

Additional file 2: Table S1. Calculated percentages based on the event counts of viable cells analyzed by flow cytometry of both BMAC groups and controls.

\section{Abbreviations}

MSC: mesenchymal stem/stromal cell(s); BM: bone marrow; BMAC: bone marrow aspirate concentrate or concentration; BMA: bone marrow aspirate; CFUF: colony forming unit-fibroblasts; WBC: white blood cell; RBC: red blood cell; (PDGF)-BB: platelet-derived growth factor; VEGF: vascular endothelial growth factor; M-CSF: macrophage colony-stimulating factor; VCAM-1: vascular cell adhesion protein 1; BMP: bone morphogenetic protein; ELISA: enzyme-linked immunosorbent assay.

\section{Authors' contributions}

JLD and RS made substantial contributions to the design, analysis, interpretation of data, and construction of the manuscript. MRD and EF made substantial contributions to the design, collection of data, analysis, and construction of the manuscript. CC, DK, JL, KB, ES, contributed substantially to the collection of data and analysis. All authors agree to be accountable for all aspects of this manuscript. All authors read and approved the final manuscript.

\footnotetext{
Author details

${ }^{1}$ Institute for Transfusion Medicine and Immunohematology, German Red Cross Blood Service Baden-Württemberg - Hessen gGmbH, Frankfurt Am Main, Germany. ${ }^{2}$ Department of Orthopedic Surgery, Stanford University School of Medicine, 450 Broadway, Redwood City, CA 94063, USA. ${ }^{3}$ CentenoSchultz Clinic, Broomfield, CO, USA. ${ }^{4}$ Institute of Transfusion Medicine and Immunology, Medical Faculty Mannheim, Heidelberg University, German
} 
Red Cross Blood Service Baden-Württemberg - Hessen gGmbH, Mannheim, Germany.

\section{Acknowledgements}

Not applicable.

\section{Competing interests}

The authors declare that they have no competing interests.

\section{Availability of data and materials}

The datasets during and/or analyzed during the current study are available from the corresponding author on reasonable request.

\section{Consent for publication}

Not applicable.

\section{Ethics approval and consent to participate}

The study was ethically approved by the Centeno-Schultz Clinic, Broomfield, $\mathrm{CO}$, USA, and material was obtained from the donors after informed consent.

\section{Funding}

The study was funded by the Department of Orthopedic Surgery, Stanford University School of Medicine and German Red Cross Blood Donor Service research funds. The funding bodies did not participate in the design of the study and collection, analysis, and interpretation of data, or in writing the manuscript.

\section{Publisher's Note}

Springer Nature remains neutral with regard to jurisdictional claims in published maps and institutional affiliations.

Received: 12 February 2019 Accepted: 28 March 2019

Published online: 08 April 2019

\section{References}

1. Smyth NA, Murawski CD, Haleem AM, Hannon CP, Savage-Elliott I, Kennedy JG. Establishing proof of concept: platelet-rich plasma and bone marrow aspirate concentrate may improve cartilage repair following surgical treatment for osteochondral lesions of the talus. World J Orthop. 2012;3(7):101-8.

2. Zhong W, Sumita Y, Ohba S, Kawasaki T, Nagai K, Ma G, et al. In vivo comparison of the bone regeneration capability of human bone marrow concentrates vs. platelet-rich plasma. PLoS ONE. 2012;7(7):e40833.

3. Hernigou P, Poignard A, Beaujean F, Rouard H. Percutaneous autologous bone-marrow grafting for nonunions. Influence of the number and concentration of progenitor cells. J Bone Joint Surg Am. 2005;87(7):1430-7.

4. Rasini V, Dominici M, Kluba T, Siegel G, Lusenti G, Northoff H, et al. Mesenchymal stromal/stem cells markers in the human bone marrow. Cytotherapy. 2013;15(3):292-306.

5. Siegel G, Kluba T, Hermanutz-Klein U, Bieback K, Northoff H, Schafer R. Phenotype, donor age and gender affect function of human bone marrow-derived mesenchymal stromal cells. BMC Med. 2013;11:146.

6. Jones $E$, Schafer R. Where is the common ground between bone marrow mesenchymal stem/stromal cells from different donors and species? Stem Cell Res Ther. 2015;6(1):143.

7. Gessmann J, Koller M, Godry H, Schildhauer TA, Seybold D. Regenerate augmentation with bone marrow concentrate after traumatic bone loss. Orthop Rev (Pavia). 2012;4(1):e14.

8. Adams SB, Lewis JS Jr, Gupta AK, Parekh SG, Miller SD, Schon LC. Cannulated screw delivery of bone marrow aspirate concentrate to a stress fracture nonunion: technique tip. Foot Ankle Int. 2013;34(5):740-4.

9. Cassano JM, Kennedy JG, Ross KA, Fraser EJ, Goodale MB, Fortier LA. Bone marrow concentrate and platelet-rich plasma differ in cell distribution and interleukin 1 receptor antagonist protein concentration. Knee Surg Sports Traumatol Arthrosc. 2016;26:333-42.
10. Hegde V, Shonuga O, Ellis S, Fragomen A, Kennedy J, Kudryashov V, et al. A prospective comparison of 3 approved systems for autologous bone marrow concentration demonstrated nonequivalency in progenitor cell number and concentration. J Orthop Trauma. 2014;28(10):591-8.

11. Hernigou P, Poignard A, Manicom O, Mathieu G, Rouard H. The use of percutaneous autologous bone marrow transplantation in nonunion and avascular necrosis of bone. J Bone Joint Surg Br. 2005;87(7):896-902.

12. Cuomo AV, Virk M, Petrigliano F, Morgan EF, Lieberman JR. Mesenchymal stem cell concentration and bone repair: potential pitfalls from bench to bedside. J Bone Joint Surg Am. 2009;91 (5):1073-83.

13. Soltan M, Smiler DG, Gailani F. A new "platinum" standard for bone grafting: autogenous stem cells. Implant Dent. 2005;14(4):322-5.

14. Doorn J, Moll G, Le Blanc K, van Blitterswijk C, de Boer J. Therapeutic applications of mesenchymal stromal cells: paracrine effects and potential improvements. Tissue Eng Part B Rev. 2012;18(2):101-15.

15. Muschler GF, Nakamoto C, Griffith LG. Engineering principles of clinical cell-based tissue engineering. J Bone Joint Surg Am. 2004;86(7):1541-58.

16. Friedlaender GE, Lin S, Solchaga LA, Snel LB, Lynch SE. The role of recombinant human platelet-derived growth factor-BB (rhPDGFBB) in orthopaedic bone repair and regeneration. Curr Pharm Des. 2013;19(19):3384-90.

17. Holmes K, Roberts OL, Thomas AM, Cross MJ. Vascular endothelial growth factor receptor-2: structure, function, intracellular signalling and therapeutic inhibition. Cell Signal. 2007;19(10):2003-12.

18. Fekete N, Gadelorge M, Furst D, Maurer C, Dausend J, Fleury-Cappellesso $S$, et al. Platelet lysate from whole blood-derived pooled platelet concentrates and apheresis-derived platelet concentrates for the isolation and expansion of human bone marrow mesenchymal stromal cells: production process, content and identification of active components. Cytotherapy. 2012;14(5):540-54.

19. Bono MR, Fernandez D, Flores-Santibanez F, Rosemblatt M, Sauma D. CD73 and CD39 ectonucleotidases in T cell differentiation: beyond immunosuppression. FEBS Lett. 2015;589(22):3454-60.

20. Belluschi S, Calderbank EF, Ciaurro V, Pijuan-Sala B, Santoro A, Mende N, et al. Myelo-lymphoid lineage restriction occurs in the human haematopoietic stem cell compartment before lymphoid-primed multipotent progenitors. Nat Commun. 2018;9(1):4100.

21. Churchman SM, Ponchel F, Boxall SA, Cuthbert R, Kouroupis D, Roshdy T, et al. Transcriptional profile of native CD271+ multipotential stromal cells: evidence for multiple fates, with prominent osteogenic and Wnt pathway signaling activity. Arthritis Rheum. 2012;64(8):2632-43.

22. Cuthbert R, Boxall SA, Tan HB, Giannoudis PV, McGonagle D, Jones E. Single-platform quality control assay to quantify multipotential stromal cells in bone marrow aspirates prior to bulk manufacture or direct therapeutic use. Cytotherapy. 2012;14(4):431-40.

23. Connolly J, Guse R, Lippiello L, Dehne R. Development of an osteogenic bone-marrow preparation. J Bone Joint Surg Am. 1989;71(5):684-91.

24. Gianakos A, Ni A, Zambrana L, Kennedy JG, Lane JM. Bone marrow aspirate concentrate in animal long bone healing: an analysis of basic science evidence. J Orthop Trauma. 2016;30(1):1-9.

25. Gobbi A, Chaurasia S, Karnatzikos G, Nakamura N. Matrix-induced autologous chondrocyte implantation versus multipotent stem cells for the treatment of large patellofemoral chondral lesions: a nonrandomized prospective trial. Cartilage. 2015;6(2):82-97.

26. Gobbi A, Karnatzikos G, Scotti C, Mahajan V, Mazzucco L, Grigolo B. Onestep cartilage repair with bone marrow aspirate concentrated cells and collagen matrix in full-thickness knee cartilage lesions: results at 2-year follow-up. Cartilage. 2011;2(3):286-99.

27. Chahla J, Mannava S, Cinque ME, Geeslin AG, Codina D, LaPrade RF. Bone marrow aspirate concentrate harvesting and processing technique. Arthrosc Tech. 2017;6(2):e441-5.

28. Dominici M, Le Blanc K, Mueller I, Slaper-Cortenbach I, Marini F, Krause D, et al. Minimal criteria for defining multipotent mesenchymal stromal cells. The International Society for Cellular Therapy position statement. Cytotherapy. 2006;8(4):315-7. 